\title{
Inhibition of autophagy via lysosomal impairment enhances cytotoxicity of fullerenol under starvation condition
}

Liyun Yang ${ }^{1,2}$, Siyu Hua ${ }^{1}$, Junpeng Fan ${ }^{3}$, Zhiqiang Zhou ${ }^{1,2}$, Guanchao Wang ${ }^{1}$, Fenglei Jiang $^{1}$, Zhixiong Xie ${ }^{3}$, Qi Xiao ${ }^{2}$, Yi Liu ${ }^{1,2 *}$

1 State Key Laboratory of Virology \& Key Laboratory of Analytical Chemistry for

Biology and Medicine (MOE), College of Chemistry and Molecular Sciences, Wuhan University, Wuhan 430072, P. R. China

2 Guangxi Key Laboratory of Natural Polymer Chemistry and Physics, College of Chemistry and Materials, Nanning Normal University, Nanning 530001, P. R. China.

3 College of Life Sciences, Key Laboratory of Analytical Chemistry for Biology and Medicine (Ministry of Education), State Key Laboratory of Virology, Wuhan University; Hubei Provincial Cooperative Innovation Center of Industrial Fermentation, Wuhan 430072, P. R. China

*Corresponding author: yiliuchem@whu.edu.cn (Y. Liu) 


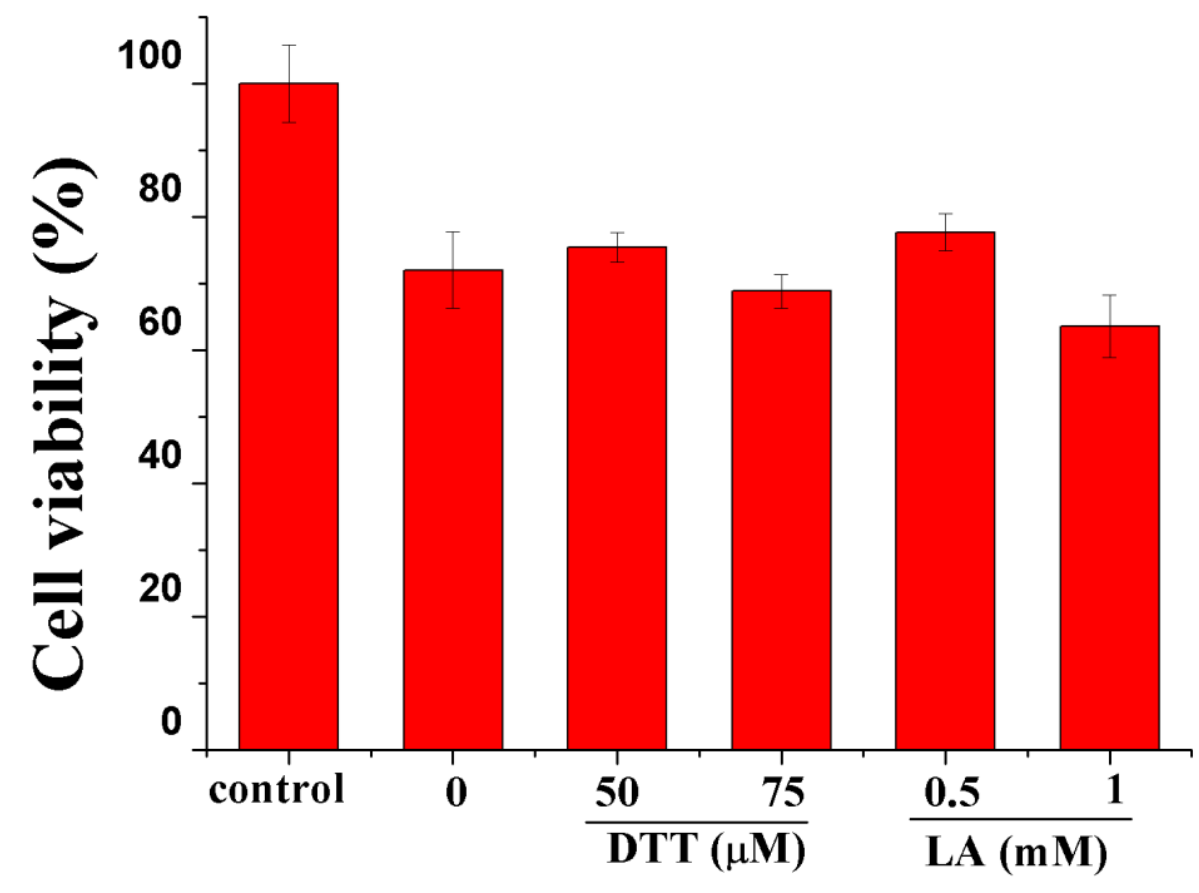

Figure S1 Fullerenol induced cell death together with DTT and LA in serum-free medium. Cells were treated with $100 \mu \mathrm{g} \mathrm{mL}^{-1}$ fullerenol together with different concentrations of DTT and LA for $24 \mathrm{~h}$ in serum-free medium.

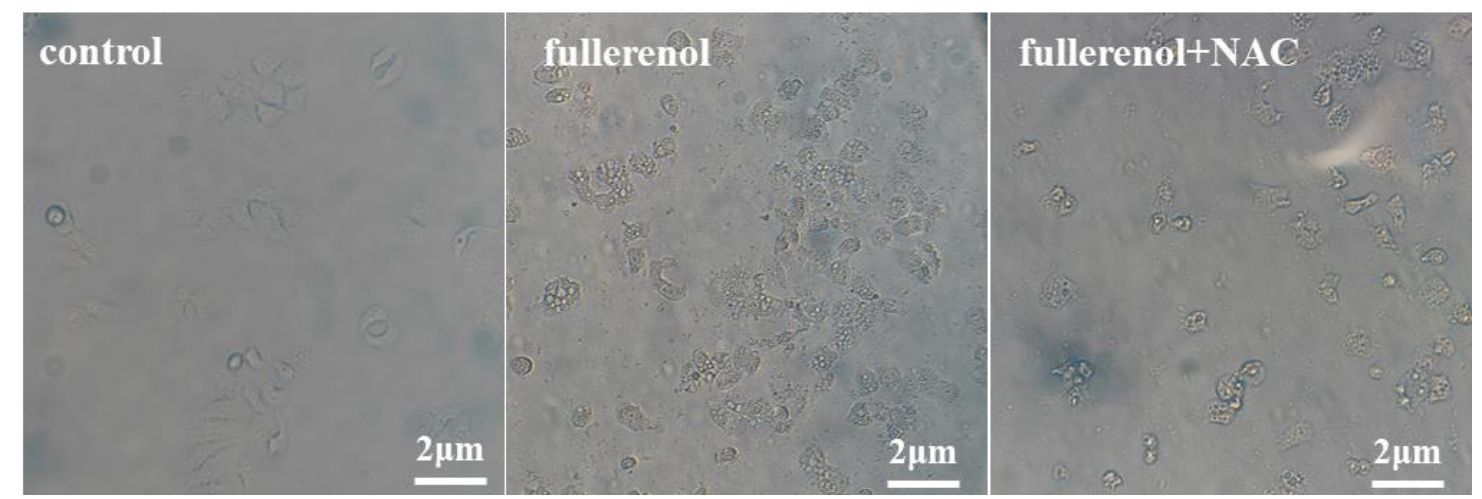

Figure S2 The microscopic picture of fullerenol-induced vacuolization at the presence of NAC in serum-free medium. Cells were treated with $100 \mu \mathrm{g} \mathrm{mL} \mathrm{m}^{-1}$ fullerenol together with different concentrations of NAC for $48 \mathrm{~h}$ in serum-free medium. 